\section{ANALYSIS AND DESIGN OF A DUAL-PORT COMPACT MICROSTRIP ANTENNA}

\author{
Manju Paulson, ${ }^{1}$ Sona O. Kundukulam, ${ }^{1}$ C. K. Aanandar,${ }^{1}$ and \\ P. Mohanan 1 \\ Centre for Research in Electromagnetics and Antennas \\ Department of Electronics \\ Cochin University of Science and Technology \\ Cochin 682 022, India
}

\section{Received 24 July 2001}

ABSTRACT: $A$ dual-port dual-polarized compact microstrip antenna for avoiding cross coupling between the two frequency bands is proposed and analyzed. This antenna offers channel isolation better than $25 \mathrm{~dB}$, and is more compact compared to a conventional rectangular patch. Analytical equations for calculating the resonant frequencies at both ports are also presented. The theoretical calculations are verified using experimental results. (c) 2002 John Wiley \& Sons, Inc. Microwave Opt Technol Lett 32: 125-127, 2002.

Key words: dual port; dual polarization; isolation; depolarization; microstrip antenna

DOI $10.1002 /$ mop. 10109

\section{INTRODUCTION}

Radar and advanced communication applications require low-profile antennas capable of dual-frequency dual-polarization operation and good isolation between the ports. Microstrip antennas of different geometries for dual polarization are available in the literature [1-3]. The severe problem with a dual-polarized satellite link is the cross coupling of cochannels, producing interference and crosstalk. These effects can be avoided using dual-port antennas, which provide excellent isolation between the ports. In this letter, we propose a compact dual-port microstrip antenna with an area reduction of $\sim 65 \%$ compared to a rectangular microstrip antenna, and an isolation better than $25 \mathrm{~dB}$ between its ports. Analytical equations for calculating the resonant frequencies at both ports are derived.

\section{ANTENNA DESIGN}

The schematic diagram of the antenna is shown in Figure 1. The structure consists of an arrow-shaped patch with an intruding triangle of height $W_{c d}$ and a protruding triangle of height $W_{c p}$ with slanted lengths $S_{1}$ and $S_{2}$ etched on a

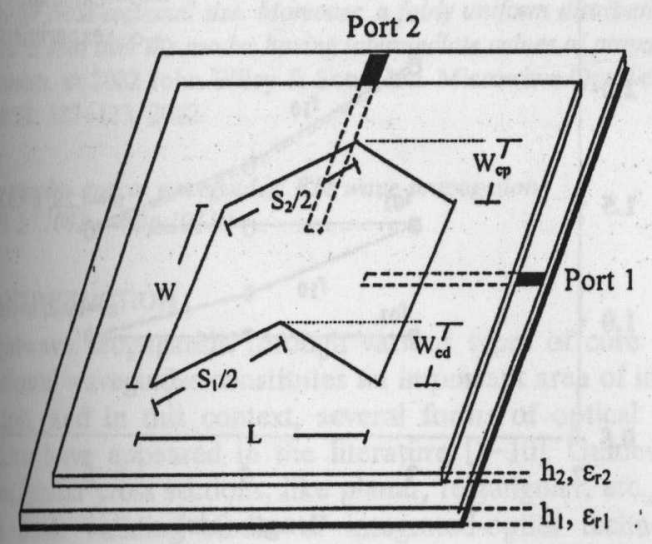

Flgure 1 Geometry of the proposed dual-port microstrip antenna dielectric substrate of thickness $h_{2}$ and dielectric const; fed by proximity coupling using two $50 \Omega$ perpendicular microstrip lines etched on a substrate of thickness $h_{1}$ and dielectric constant $\epsilon_{r 1}$.

A typical antenna structure of length $L=0.04 \mathrm{~m}$, width $W=0.06 \mathrm{~m}, W_{c d}=0.01 \mathrm{~m}$, and $W_{c p}=0.01 \mathrm{~m}$ is etched on a substrate of thickness $h_{1}=0.0016 \mathrm{~m}$ and $\varepsilon_{r 1}=4.28$. Two $50 \Omega$ microstrip feedlines are fabricated on a similar substrate kept below the patch to provide electromagnetic coupling.

\section{RESONANT FREQUENCY CALCULATION}

The standard equations for computing the resonant frequency of a rectangular patch antenna are modified to take into account the effect of the intruding and protruding lengths $W_{c d}$ and $W_{c p}$. The line extension factor and the effective resonating lengths of the two modes are obtained by the following empirical relations. Here, $h$ used in the equations is the effective thickness $h=h_{\text {eff }}\left(h_{\text {eff }}=h 1+h 2\right)$ and the dielectric constant $\varepsilon_{r}=\varepsilon_{r 1}=\varepsilon_{r 2}$.

For Port 2 ,

$$
\begin{gathered}
f_{10}=\frac{c}{2\left(S_{\mathrm{eff}}+2 \Delta l_{1}\right) \sqrt{\varepsilon_{1}}} \\
\varepsilon_{1}=\frac{\varepsilon_{r}+1}{2}+\frac{\varepsilon_{r}-1}{2}(1+12 h / W)^{-1 / 2} \\
\Delta l_{1}=\frac{0.412 h\left(\varepsilon_{1}+0.3\right)(W / h+0.258)}{\left(\varepsilon_{1}-0.258\right)(W / h+0.8)} .
\end{gathered}
$$

For Port 1,

$$
f_{01}=\frac{c}{2\left(W_{\text {eff }}+2 \Delta l_{2}\right) \sqrt{\varepsilon_{2}}}
$$

where $s_{2}$ and $\Delta l_{2}$ are calculated by replacing $W$ by $S$ in Eqs. (2) and (3), respectively, with $S=\left(S_{1}+S_{2}\right) / 2$

$S_{\text {eff }}$ and $W_{\text {eff }}$ are calculated as follows.

For $(L<W)$,

$$
\left.\begin{array}{rl}
S_{\text {eff }}= & S_{1}-(0.0001 / L)+0.01 W \\
& -0.68\left(W_{c d}-0.01\right)-0.03\left(W_{c p}-0.01\right) \\
W_{\text {eff }}= & W+0.58 W_{c p}-0.43 W_{c d} \quad \text { for } W_{c d} / W \leq 0.5 \\
S_{\text {eff }}= & 0.5\left(S_{1}+L\right)+0.4 W_{c d}-0.175 W-0.03\left(W_{c p}-0.01\right) \\
W_{\text {eff }}= & 0.78 W+0.025 W_{c d}+0.49 W_{c p}
\end{array}\right\}
$$

for $W_{c d} / W>0.5$.

For $(L \geq W)$

$$
\begin{aligned}
& S_{\text {eff }}=S_{1}+2.3(L-2 W-0.0046 / L) W_{c d} \\
& +0.00006 / L-0.1\left(W_{c p}-0.01\right), \quad \text { for } W_{c d} / W<1 \\
& W_{\text {eff }}=W+0.58 W_{c p}-0.43 W_{c d}+0.0023(L-W) / W \text {, } \\
& \text { for } W_{c d} / W \leq 0.5 \\
& W_{\text {eif }}=0.78 W+0.025 W_{c d}+0.49 W_{c p}+0.0025 W_{c d} / W \\
& +0.17(L-W-0.01), \quad \text { for } W_{c d} / W>0.5
\end{aligned}
$$




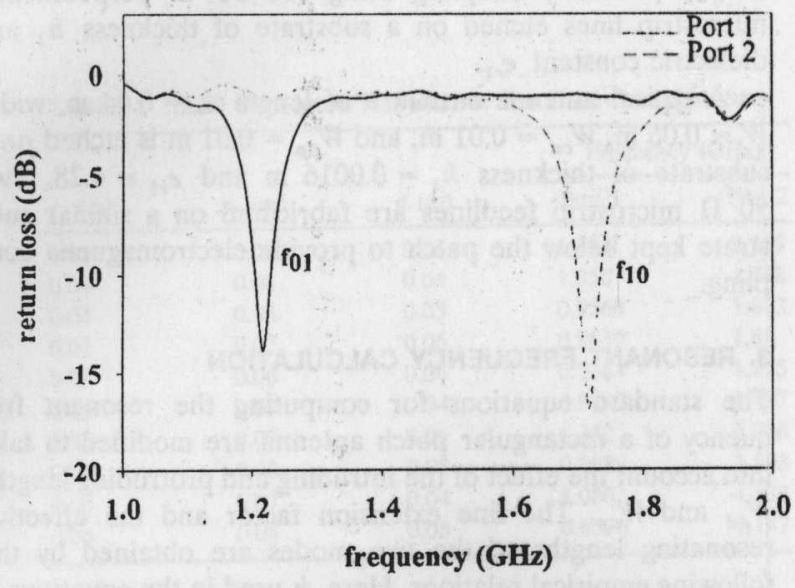

Figure 2 Measured return loss against frequency for two ports

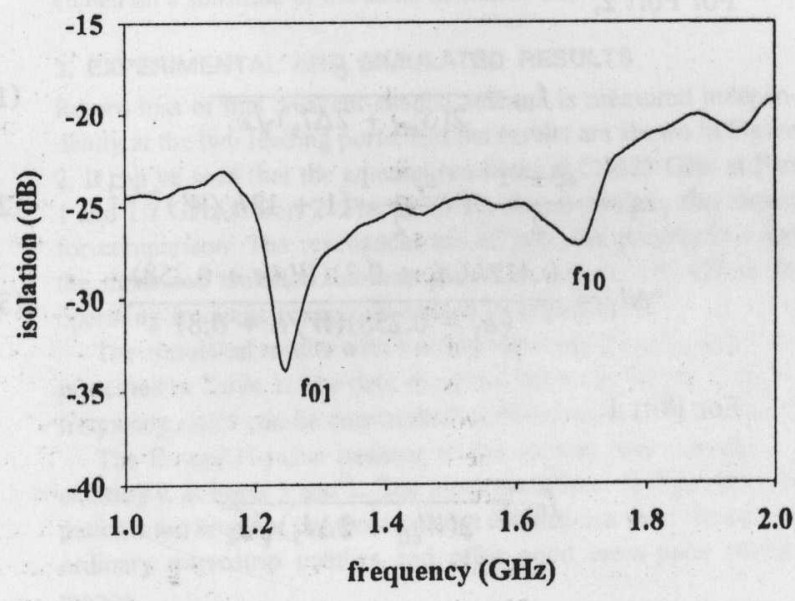

Figure 3 Measured isolation between ports 1 and 2

\section{EXPERIMENTAL AND THEORETICAL RESULTS}

The measured return loss against frequency for the two ports are shown in Figure 2. Figure 3 shows the isolation between the two ports, and Figure 4 shows their transmission characteristics. From these figures, it is clear that the isolation between the ports is better than $25 \mathrm{~dB}$, which implies very small cross coupling. The $E$-plane and $H$-plane patterns for

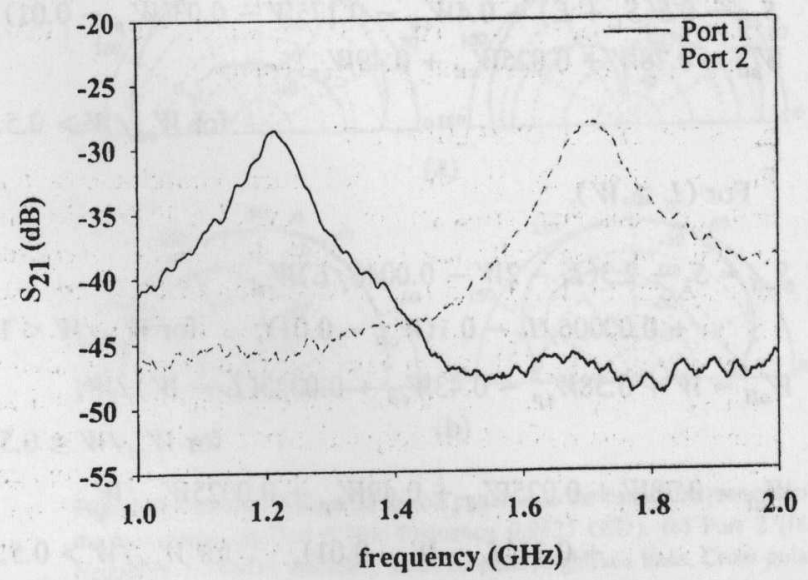

Figure 4 Measured transmission characteristics against frequency at two ports

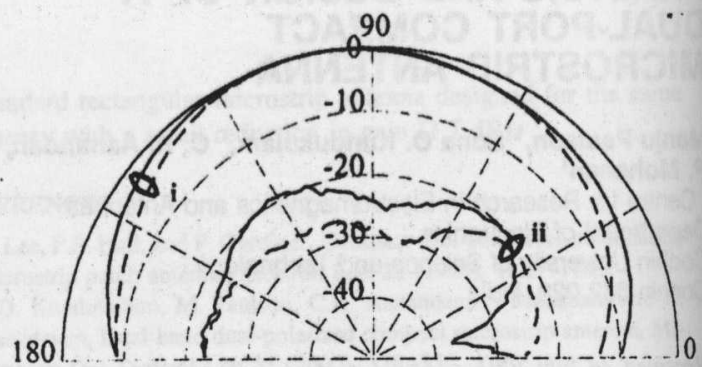

(a)

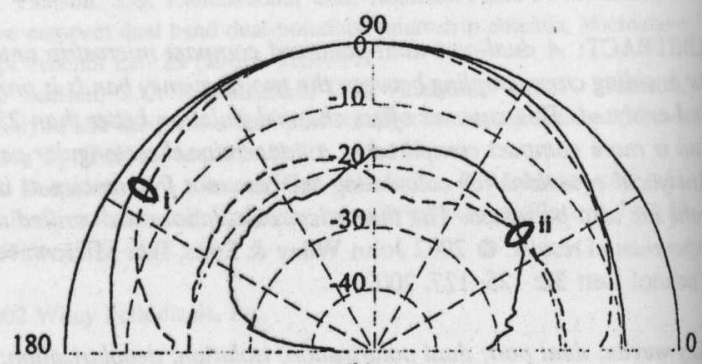

(b)

Figure $5 E$-plane and $H$-plane radiation patterns. (a) Port 1 fre quency $\left(f_{01}=1.2175 \mathrm{GHz}\right)$. (b) Port 2 frequency $\left(f_{10}=1.7175 \mathrm{GHz}\right.$ ). i) Copolarization. ii) Cross polarization. $-E$-plane, $----H$-plane

the frequencies 1.2175 and $1.7175 \mathrm{GHz}$ are shown in Fig ure 5 . The cross-polar performance for both frequencies is found to be better than $20 \mathrm{~dB}$.

The theoretical variation of the two resonant frequencies at port $1\left(f_{01}\right)$ and port $2\left(f_{10}\right)$ with $L$ for different heights $h$ and permittivities $\varepsilon_{r}$ are shown in Figure 6 . The experimental curves are also given in the same figure to validate the computation. In all of these cases, the theoretical results are in good agreement with experimental values, with a maximum error of less than $2 \%$.

\section{CONCLUSION}

A compact dual-port antenna for avoiding channel cross coupling in satellite communication is presented. The proposed antenna offers a greater size reduction compared to a standard rectangular patch and improved performance characteristics compared to the other compact patches. Analytica

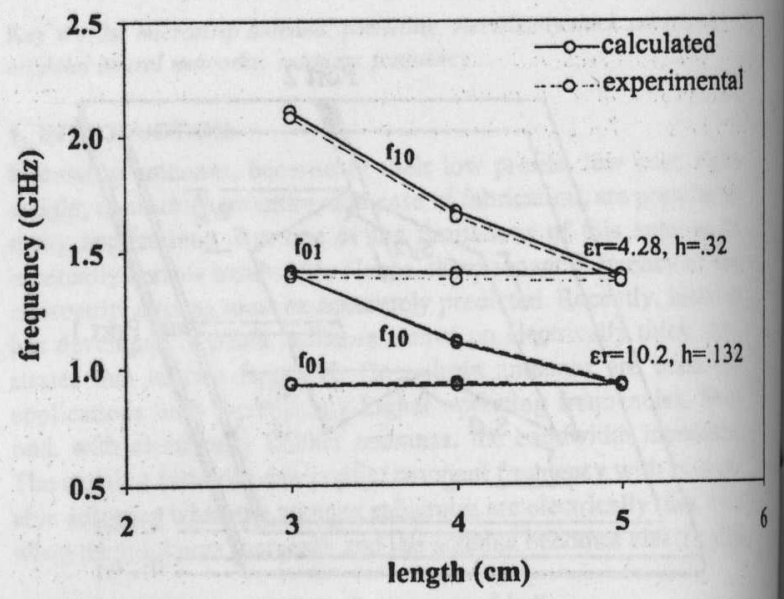

Figure 6 Variation of $f_{10}$ and $f_{01}$ with length $L$ for different $\varepsilon_{1}$ and $h$ values for the dual-port antenna $\left(W=0.05 \mathrm{~m}, W_{c d}=0.01 \mathrm{~m}\right.$ $W_{c p}=0.01 \mathrm{~m}$ ) 
equations developed here will be helpful in designing an antenna with different frequency ratios for different applications.

\section{REFERENCES}

1. S.O. Kundukulam, M. Paulson, C.K. Aanandan, P. Mohanan, and K. Vasudevan, Dual-band dual-polarized compact microstrip antenna, Microwave Opt Technol Lett 25 (2000), 328-330.

2. M. Paulson, S.O. Kundukulam, C.K. Aanandan, and P. Mohanan, A new compact dual-band dual-polarized microstrip antenna, Microwave Opt Technol Lett 29 (2001), 315-317.

3. M. Deepukumar, J. George, C.K. Aanandan, P. Mohanan, and K.G. Nair, Broadband dual frequency microstrip antenna, Electron Lett 32 (1996), 1531-1532.

c) 2002 John Wiley \& Sons, Inc. 